\title{
The fibroproliferative response in acute respiratory distress syndrome: mechanisms and clinical significance
}

\author{
Ellen L. Burnham¹, William J. Janssen ${ }^{1,2}$, David W.H. Riches ${ }^{1,3}$, Marc Moss ${ }^{1}$ and \\ Gregory P. Downey ${ }^{1,2,3}$
}

\author{
Affiliations: \\ 'Division of Pulmonary Sciences and Critical Care Medicine, Dept of Medicine, University of Colorado Health \\ Sciences Center, Aurora, CO, \\ ${ }^{2}$ Division of Pulmonary, Critical Care, and Sleep Medicine, Dept of Medicine, National Jewish Health, Denver, \\ $\mathrm{CO}$, and \\ ${ }^{3}$ Program in Cell Biology, Dept of Pediatrics, National Jewish Health, Denver, CO, USA.
}

Correspondence: G.P. Downey, Office of Academic Affairs k701b, National Jewish Health, 1400 Jackson Street, Denver, C0 80206, USA. E-mail: downeyganjhealth.org

ABSTRACT Acute respiratory distress syndrome (ARDS) continues to be a major healthcare problem, affecting $>190000$ people in the USA annually, with a mortality of $27-45 \%$, depending on the severity of the illness and comorbidities. Despite advances in clinical care, particularly lung protective strategies of mechanical ventilation, most survivors experience impaired health-related quality of life for years after the acute illness. While most patients survive the acute illness, a subset of ARDS survivors develops a fibroproliferative response characterised by fibroblast accumulation and deposition of collagen and other extracellular matrix components in the lung.

Historically, the development of severe fibroproliferative lung disease has been associated with a poor prognosis with high mortality and/or prolonged ventilator dependence. More recent studies also support a relationship between the magnitude of the fibroproliferative response and long-term health-related quality of life. The factors that determine which patients develop fibroproliferative ARDS and the cellular mechanisms responsible for this pathological response are not well understood.

This article reviews our current understanding of the contribution of pulmonary dysfunction to mortality and to quality of life in survivors of ARDS, the mechanisms driving pathological fibroproliferation and potential therapeutic approaches to prevent or attenuate fibroproliferative lung disease.

@ERSpublications

Despite protective ventilation, a subset of ARDS survivors develop lung fibrosis contributing to poor quality of life http://ow.ly/q8xCo 


\section{Diminished quality of life in acute respiratory distress syndrome survivors}

Since the initial description of the acute respiratory distress syndrome (ARDS) by AsHBAUGH et al. in 1967 [1], the overall mortality associated with the disorder has decreased [2,3]. Multicentre randomised clinical trials have been a major driver of this improvement, fostering advances such as the use of positive endexpiratory pressure (PEEP) [4], low tidal volume ventilation [5] and conservative fluid management [6]. Although these interventions have resulted in improved survival and reduced time spent on mechanical ventilation, it is increasingly recognised that a substantial proportion of ARDS survivors continue to suffer from reduced health-related quality of life (HRQoL) that lasts for months to years [7-12]. While recent investigations have drawn attention to extrapulmonary complications of ARDS, such as depression and neuromuscular weakness, residual pulmonary dysfunction has been largely discounted as a significant contributing factor to diminished HRQoL. Indeed, the prevailing opinion among ARDS experts in the current era of lung protective mechanical ventilation is that the prevalence of persistent pulmonary impairment in ARDS survivors has decreased. However, perusal of available data suggests that pathological fibroproliferation in the lung continues to play a critical role in both short-term and longer-term outcomes in ARDS patients. Understanding the clinical relevance of profibrotic activity in ARDS, and predicting which patients are at risk of the development of excessive fibroproliferation, will pave the way for the identification of novel therapies that can target specific pathways involved in maintenance and restoration of normal lung architecture.

\section{Persistent pulmonary dysfunction in the low tidal volume era}

The most current data assessing the prevalence of pulmonary dysfunction among ARDS survivors arise from four independent cohorts in which pulmonary function tests (PFTs) were performed 6 months to 5 years after intensive care unit (ICU) discharge [7-13]. These studies each demonstrated median values for forced vital capacity (FVC), forced expiratory volume in $1 \mathrm{~s}$ (FEV1), total lung capacity (TLC) and diffusion capacity of the lung for carbon monoxide (DLCO) that were within the normal range (i.e. $>80 \%$ predicted). However, for each parameter, values in the lowest quartile were consistently below normal $[8,10,12,13]$. Notably, in one study over half of the survivors had impairments in at least one of these parameters [11]. Importantly, decrements in lung function changed little after the first year, and even 5 years after discharge, subjects in the lowest quartile of one study displayed a persistently reduced FEV1, FVC, TLC, residual volume and DLCO [9]. Nevertheless, the association between these physiological abnormalities and parenchymal lung disease remains poorly defined, since PFTs may be influenced by fibrotic lung disease as well as by neuromuscular weakness, the latter being increasingly recognised as a cause of diminished HRQoL [7].

To assess the clinical relevance of physiological abnormalities identified in ARDS survivors, associations between PFTs and HRQoL measured via standardised questionnaires have been explored [10, 12]. HEYLAND et al. [10] observed a modest association between lower FVC and FEV1 and poorer scores on the physical component subtotal of the 36-item short form health survey (SF-36) ( $\rho>0.5$ for comparisons) as well as the St George's Respiratory Questionnaire (SGRQ) ( $\rho>0.4$ for comparisons). Similar associations between FVC, FEV1, DLCO and TLC and physical function scores on the SF-36 ( $\rho>0.4$ for comparisons) were reported by ORME et al. [12]. However, in most ARDS survivor investigations, respiratory-specific questionnaires such as the SGRQ [14] have been performed much less frequently than questionnaires that assess global health and wellbeing, such as the SF-36 [15], where responses may be influenced by respiratory as well as neuromuscular symptoms. Moreover, the fact that a sizable percentage of ARDS survivors have a significantly diminished $6 \mathrm{~min}$ walk distance $[8,9,11]$, further suggests that both neuromuscular impairment and pulmonary dysfunction may contribute to impairments in HRQoL. Therefore, to define the impact of residual pulmonary impairment among ARDS survivors on HRQoL, respiratory-specific assessment that confirms pulmonary pathophysiological abnormalities is necessary.

Computed tomographic (CT) scanning of the lungs of ARDS survivors often demonstrates reticular infiltrates (fig. 1) that may persist for months to years after discharge from the ICU [9, 11, 16-18], although these changes were not observed in all studies [13]. Surprisingly, although the extent of reticular involvement in ARDS survivors has been associated with specific factors implicated in the pathogenesis of fibrotic lung disease, such as high airway pressures, hyperoxia and longer duration of mechanical ventilation $[16,17]$, few studies have systematically examined chest CT scans in association with physiological function among ARDS survivors $[9,11,13,18]$. Published studies that have included both PFT and chest CT scans emphasise that persistent radiographical abnormalities are generally not severe in the majority of patients and have therefore been considered to be clinically unimportant $[9,11,13]$. However, most studies have not attempted to correlate chest CT scan data objectively with outcomes (including PFTs and HRQoL measurements). Utilising prospectively collected high resolution CT scans (HRCT), PFTs and HRQoL data from a randomised clinical trial in patients with ARDS [19], our group recently reported that the extent of 


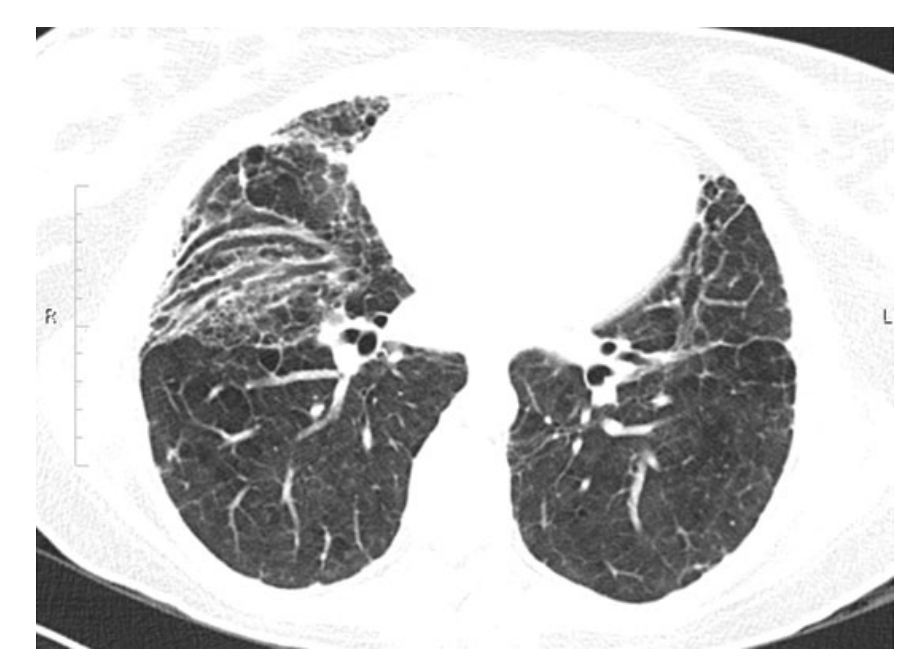

FIGURE 1 High-resolution computed tomography image of the base of the lungs of an actue respiratory distress syndrome survivor demonstrating persistent reticular changes in the lungs and traction bronchiectasis 18 months after the acute illness.

persistent reticular abnormalities on HRCT correlated with reduced pulmonary-specific HRQoL as assessed by SGRQ surveys obtained at 180 days [18]. Notably, these HRCT abnormalities correlated with physiological features of restrictive lung disease, including low TLC, FVC and DLCO. As in prior survivor studies, $\sim 25 \%$ of ARDS survivors had physiological evidence of restrictive pulmonary physiology at 180 days. Furthermore, patients who displayed features of restrictive physiology at 180 days exhibited reticular infiltrates on HRCT 14 days after diagnosis, and their CT scans remained significantly more affected by reticulation over time compared to patients who did not develop restrictive physiology [18]. These data, collected from almost 100 patients, provide additional evidence to support the concept that the lungs of a subset of ARDS survivors have persistent fibrotic changes that, in many cases, correlate with diminished HRQoL and restrictive pulmonary physiology. In future studies, systematic assessment of ARDS patients using pulmonary-specific metrics will be necessary to establish more precisely the prevalence of clinically significant pulmonary impairment.

\section{Basic mechanisms driving fibroproliferation in ARDS}

Our understanding of the basic mechanisms driving the pathological fibroproliferative response in ARDS has evolved substantially over the last two decades (fig. 2). ARDS is thought to develop following injury to, and disruption of, the alveolar-capillary barrier with persistent injury to the alveolar epithelium [20-22]. This, in turn, sets in motion a series of events including the exudation of plasma into the alveolar spaces, which, together with impaired alveolar fluid clearance, leads to alveolar oedema. Activation of the coagulation system and production of proinflammatory cytokines and chemokines leads to an influx of neutrophils, monocytes/macrophages and lymphocytes into the lung associated with injury to the alveolocapillary membrane $[20,23]$. During the acute inflammatory phase of ARDS, unregulated release of potent cytotoxic mediators from infiltrating leukocytes, including reactive oxygen and nitrogen species and proteolytic enzymes such as elastase and matrix metalloproteinases (MMPs), results in injury to pulmonary endothelial and epithelial cells and, if severe, damages the scaffolding of the lung [23-26]. The combination of ongoing injury and failure to repair this damage in a timely manner are major factors that contribute to the pathological fibroproliferative response [27]. While the inflammatory response resolves in a timely manner in most patients, in others there is robust and persistent accumulation of macrophages, fibrocytes, fibroblasts and myofibroblasts in the alveolar compartment, leading to excessive deposition of fibronectin, collagens I and III and other components of the extracellular matrix. This is accompanied by an imbalance between profibrotic (e.g. transforming growth factor (TGF)- $\alpha$ and $-\beta$, interleukin- $\beta$, platelet-derived growth factor and lysophosphatidic acid) and antifibrotic (e.g. prostaglandin E2, keratinocyte growth factor and hepatocyte growth factor) mediators that may drive this fibroproliferative response [28-36]. As in other fibrotic disorders of the lung, the origin of the fibroblasts that accumulate in the lung in fibroproliferative ARDS remains uncertain and controversial [20, 37-40]. Lung-resident (interstitial) fibroblasts, the mesenchymal differentiation of lung epithelial cells and pericytes, as well as emigration of bone marrow derived fibrocytes have all been implicated as potential sources of the increased lung fibroblasts [37, 40, 41].

The initial inflammatory injury to the cellular and structural elements of the lung may be amplified by damage from shear forces imparted by mechanical ventilation $[42,43]$, underscoring the importance of lung protective ventilation strategies to prevent ongoing epithelial injury that may drive fibroproliferation $[5,44]$. Severe damage to the underlying structural elements of the lung, including the vasculature and the extracellular matrix scaffolding of the lung, makes repair of the delicate pulmonary architecture much more 


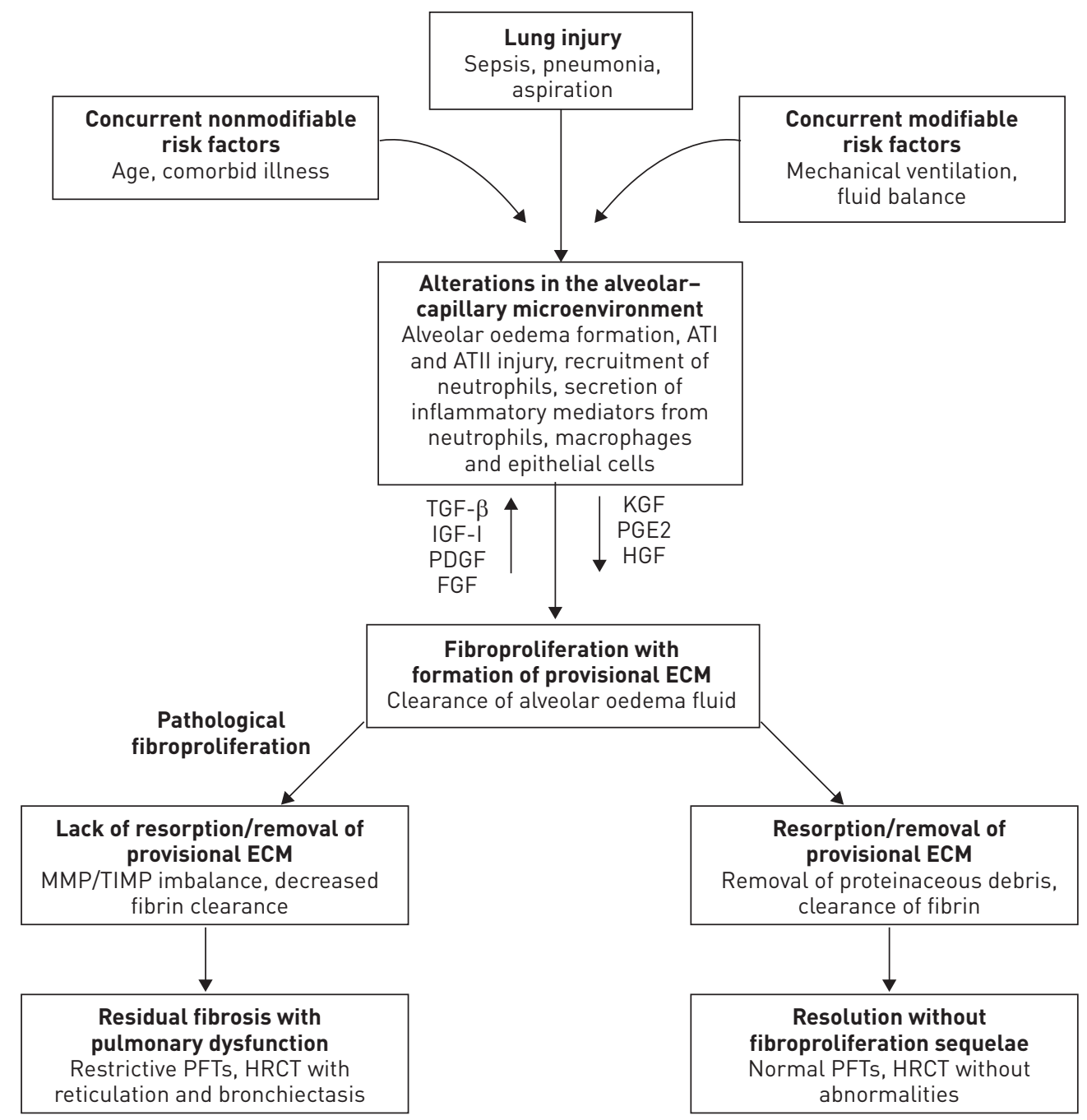

FIGURE 2 Mechanisms driving fibroproliferation in acute respiratory distress syndrome. Characteristic alterations related to lung injury evolve over time, and may be influenced by a variety of environmental and patient-specific factors. In response to alveolar-capillary injury, a provisional extracellular matrix (ECM) is ultimately formed to promote repair, and proteinaceous pulmonary oedema fluid is cleared from the alveolar space. The provisional ECM largely resolves after restoration of lung architecture; however, a subset of patients will not clear the provisional ECM and exhibit evidence of exuberant fibroproliferation with fibrosis lasting from months to years after the acute event. Bold type represents major "activity" at this time point. AT: angiotensin; TGF: transforming growth factor; IGF: insulin-like growth factor; PDGF: platelet-derived growth factor; FGF: fibroblast growth factor; KGF: keratinocyte growth factor; PGE: prostaglandin E; HGF: hepatocyte growth factor; MMP: matrix metalloproteinases; TIMP: tissue inhibitor of metalloproteinase; PFT: pulmonary function test; HRCT: high-resolution computed tomography.

difficult and predisposes to pathological fibroproliferation. Stated in another way, if the "blueprint" specifying details of the intricate architecture of the lung is "lost in the ruins", orderly repair is likely to fail.

One key component of the infrastructure in the lung that is damaged in ARDS is the vasculature [45, 46]. Vascular lesions that have been recognised in ARDS include endothelial injury, increased microvascular permeability, thrombotic, fibroproliferative and obliterative changes that correlate temporally with histological evidence of diffuse alveolar damage [21, 22, 46, 47]. These macro- and microvascular alterations must be repaired in order to restore lung function. Furthermore, failure to repair the vasculature may contribute to the severity and persistence of lung injury $[45,46]$ and to disorganised repair. Indeed, there is experimental evidence that excessive or unregulated angiogenic responses, mediated by angiogenic cytokines and growth factors, such as macrophage inflammatory protein-2, angiopoietin 2 and vascular endothelial growth factor (VEGF), may contribute to the initial injury and drive the fibroproliferative response [48-50]. In translational studies, pulmonary levels of VEGF are decreased in the early stages of ARDS [51-53], but then subsequently increase in a fashion that tracks the recovery from lung injury [52]. 
Excess levels of circulating angiopoietin 2 occur early in ARDS and may contribute to vascular leak as well as distort the microvascular architecture of the lung [54].

\section{Evidence of fibroproliferative activity in early ARDS in translational studies}

Whereas large-scale studies of pulmonary function in long-term survivors of ARDS remain sparse, ample evidence for early fibroproliferative activity in the lungs of ARDS patients has been reported for decades. Since lung biopsies are not performed in the majority of patients, investigations utilising bronchoalveolar lavage have provided most of the available information. Pioneering work by several groups in the 1990s demonstrated evidence of robust collagen synthesis (reflected by high levels of N-terminal peptide of type III procollagen) in the lungs of ARDS patients as early as $72 \mathrm{~h}$ after the onset of disease [55-57]. Interestingly, the presence of procollagen peptide III correlated with risk for fatal outcome [55, 57]. Histological assessment of the lungs of ARDS patients, although limited, clearly demonstrates that fibroproliferation is present early in a substantial proportion of patients. For example, BuLPA et al. [58] reported that in a series of 11 immunocompetent patients who met the American-European Consensus Conference (AECC) criteria for ARDS and who underwent transbronchial biopsy a median of 10 days after intubation, pathological evidence of fibroproliferation was observed in every patient. PATEL et al. [59] reviewed the records of patients who met AECC criteria for ARDS and who underwent an open lung biopsy. Of the 57 patients who were biopsied on average 3 days after intubation ( 7 days after hospital admission), 23 had a pathological evidence of diffuse alveolar damage, supporting the diagnosis of ARDS, and 18 (78\%) out of 23 were considered to be in the fibroproliferative phase of ARDS at the time of biopsy [59]. The largest prospective study to date of surgical lung biopsies from ARDS patients included 100 patients hospitalised between 1996 and 2003 [60]. Samples were obtained a median 7 days after ARDS diagnosis and 11 days after the initiation of mechanical ventilation. Fibrosis was noted in 53 out of 100 patients, and was associated with evidence of infection in 29 (55\%) out of 53. Additional studies that used lung biopsy to study ARDS patients have demonstrated that profibrotic mediators including TGF- $\beta_{1}$ and insulin-like growth factor-I are expressed during (and probably contribute to) the fibroproliferative response in ARDS $[36,61]$, thus validating the experimental studies discussed earlier.

Despite the widespread adoption of low tidal volume ventilator strategies [5], investigators continue to report early fibroproliferative activity in lungs of ARDS patients. For example, MARSHALL et al. [62] reported that $24 \mathrm{~h}$ after the onset of illness, N-terminal procollagen peptide III levels were significantly greater in the bronchoalveolar lavage fluid (BALF) and serum of ARDS patients than controls. Moreover, BALF from ARDS patients induced significantly greater proliferation of cultured human lung fibroblasts than BALF from controls [62]. BALF from ARDS patients potently induces $\alpha$-smooth muscle actin expression and procollagen-1 promoter activity in cultured human lung fibroblasts via activation of the TGF- $\beta$ receptors $[63,64]$. Importantly, TGF- $\beta_{1}$ concentrations are markedly increased in BALF as early as $24 \mathrm{~h}$ after the onset of ARDS $[4,36]$, and when elevated in serum are associated with lower survival in ARDS patients with septic shock [65]. Two recent studies have focused on potential cellular mediators of fibrosis, including mesenchymal cells with fibroblast-like properties (alveolar fibroblasts or fibrocytes) that have been isolated by bronchoalveolar lavage of ARDS patients within the first 7-14 days after diagnosis [66, 67]. In both studies, the numbers of these cells in BALF were greater in ARDS patients than in ventilated controls. In ARDS patients with a positive fibroblast culture, concentrations of BALF procollagen I were significantly higher [66], but neither procollagen I nor other markers of fibroproliferation correlated with the fibrocyte number [67]. However, in multivariable analyses, alveolar fibrocyte percentage was independently associated with increased mortality in patients with ARDS, accounting for potential effects of age, concomitant malignancy and severity of illness (calculated using sequential organ failure assessment scores).

\section{Identification of excessive fibroproliferation may help guide clinical decisions}

Importantly, while excessive fibroproliferation is clearly detrimental, an "appropriate" fibroproliferative response may have beneficial consequences in guiding lung repair, for example by serving as a scaffold and providing a reservoir for nutrients and growth factors [68] for the regenerating alveolar epithelium. Thus, markers that distinguish "appropriate" fibroproliferative activity from excessive and potentially harmful fibroproliferation are desperately needed. Indeed, identifying ARDS patients with aberrant fibroproliferative activity may prove to be helpful clinically. For example, such identification could aid in prognostic discussions with patients and their family members. Furthermore, measurement of specific profibrotic mediators may assist in predicting which patients are most likely to benefit from targeted antifibroproliferative therapies, and may help to monitor the clinical response of such agents. Characterisation of fibroproliferative activity in acutely ill ARDS patients could involve measuring mediators in BALF, blood or urine, radiological assessments or combinations thereof. These results would need to be interpreted in light of the patient's history and clinical course. For example, mechanical 
ventilation parameters [16, 17] and specific risk factors underlying ARDS development [69] have been associated with a more protracted clinical course and subsequent fibrotic lung disease.

Bronchoscopy with bronchoalveolar lavage in the critically ill patient with ARDS is relatively safe [70], may be performed at the bedside and provides information regarding fibroproliferative activity in the airspaces. The optimal timing to assess fibroproliferative activity in newly diagnosed ARDS is uncertain. Furthermore, given the spectrum of illness of ARDS, not all patients may be able to tolerate bronchoscopy safely at a consistent time point. Moreover, bronchoalveolar lavage does not always provide a reproducible yield, so it is difficult to establish specific concentrations or cut-points for prognostic assays that rely exclusively on bronchoalveolar lavage. For these reasons, measurement of a systemic biomarker in the bloodstream that reflects fibroproliferative activity in lungs would be preferable to identify aberrant fibroproliferation in ARDS. In idiopathic pulmonary fibrosis (IPF), systemic biomarkers have been identified that reflect disease activity and prognosis and may be predictive of response to therapy [71, 72]. Many of these biomarkers have a plausible role in the pathogenesis of fibrotic disease and are therefore potential therapeutic targets. Notably, certain IPF-associated biomarkers also correlate with outcomes in ARDS, including surfactant protein-D and macrophage chemotactic protein-1 [73-75]. Interestingly, circulating fibrocytes, which may have prognostic utility in IPF, have been identified in the blood of patients with ARDS [71]. Additional investigations will be necessary to establish whether these circulating factors are indeed causally associated with pathological fibroproliferative activity in lung.

HRCT scans of the chest are routinely used to diagnose and manage patients with idiopathic interstitial pneumonias, including IPF. In relation to ARDS, GATTINONI et al. [76] have demonstrated the utility of standard (low resolution) CT scanning in tailoring PEEP and tidal volume in ARDS patients. Contemporaneous work by ICHIKADO et al. [77] has suggested an additional role for HRCT in early ARDS prognostication. These investigators retrospectively examined HRCTs obtained an average of 7 days after ARDS diagnosis, and using a previously established scoring system [77], determined that more extensive lung involvement, particularly with bronchiolectasis or bronchiectasis, was associated with increased mortality, longer time on mechanical ventilation, decreased organ failure-free days and barotrauma [78]. More recently, this same group reported that scores from HRCT scans obtained $24 \mathrm{~h}$ after ARDS diagnosis were associated with clinical outcomes, including ventilator- and organ failure-free days, as well as 60- and 90-day mortality [79]. Higher scores in this series of patients were again driven by the occurrence of traction bronchiolectasis or bronchiectasis [79]. A separate group has confirmed the utility of HRCT obtained during the acute phase of pathologically confirmed diffuse alveolar damage, and found that increased radiological lung involvement, as well as traction bronchiectasis, were associated with death [80]. Finally, published studies of the $2009 \mathrm{H} 1 \mathrm{~N} 1$ epidemic indicate that HRCT is similarly useful in predicting mortality and long-term morbidity in patients with ARDS from influenza A $[69,81]$. Our group's recent observations complement and add to these investigations and suggest that HRCT performed early in the course of ARDS may prognosticate functional impairment in survivors months after discharge [18]. Questions that remain to be answered are how to define objectively chest HRCT radiological patterns that most accurately reflect active fibroproliferation and the optimal time at which HRCTs will be most clinically useful.

\section{Potential therapies for pathological fibroproliferation in ARDS}

Notably, no pharmacological agents that specifically target fibroproliferation are currently available for treatment of ARDS. Based on data from research conducted in the 1990s, randomised clinical trials of corticosteroids in patients with nonresolving ARDS were conducted. In these studies, corticosteroids were administered 1 week after the onset of illness to decrease inflammation and modulate fibroproliferative activity with the goal to improve short-term outcomes [82, 83]. In these investigations, no mortality benefit was observed, but shortened time on mechanical ventilation was reported in secondary analyses. Moreover, in the latter study, increased mortality was noted in ARDS patients who were treated with corticosteroids after 14 days or who had BALF procollagen peptide III levels below the group median. In a subsequent study of 91 patients, early $(72 \mathrm{~h})$ treatment with corticosteroids in severe ARDS was associated with a shortened duration of mechanical ventilation [84]. As a whole, the available data suggest that corticosteroids do not favourably impact ARDS survival but may minimise ventilatory requirements. The use of corticosteroids in ARDS remains of unproven benefit and the source of controversy.

Other considerations for therapies to mitigate aberrant fibroproliferative activity in ARDS include agents being developed to treat pulmonary fibrosis, such as tyrosine kinase inhibitors [85], peptides directed at extracellular matrix proteins and their cellular receptors [86] and molecules directed at TGF- $\beta$ and the profibrotic pathways regulated by this pleiotropic growth factor (table 1). The rapidly increasing knowledge of TGF- $\beta$ signalling pathways and how they control cell proliferation, apoptosis, inflammation and fibrosis is being exploited to develop novel therapeutic approaches to treat human diseases such as cancer [87-89]. As many of these TGF- $\beta$ signalling pathways, including canonical (Smad-dependent) and non-canonical 
TABLE 1 Molecular targets to limit pathological fibroproliferation

\section{Tyrosine kinase inhibitors Src kinase inhibitors}

Histone deacetylase inhibitors

Monoclonal antibodies/blocking peptides

Activating peptides
Inhibit receptor signalling

Inhibit profibrotic signalling downstream of receptors

Prevent nuclear translocation and DNA binding of Smads

Directly bind to growth factors or block receptor ligation binding

Inhibit synthesis of matrix proteins
TGF- $\beta$, PDGF, VEGF, FGF, angiopoietin TGF- $\beta$, PDGF

TGF- $\beta$, PDGF

TGF- $\beta$, PDGF, IGF-1, FGF, angiopoietin Relaxins, endostatin

TGF: transforming growth factor; PDGF: platelet-derived growth factor; VEGF: vascular endothelial growth factor; FGF: fibroblast growth factor: IGF: insulin-like growth factor.

(e.g. phosphatidylinositide (PI)3 kinase, Akt and p38 mitogen-activated protein kinase) pathways, also drive fibroproliferative responses (which incidentally also drive the stromal responses associated with tumours), these agents could conceivably be used in the treatment of fibroproliferative ARDS.

\section{Summary and conclusions}

Despite major advances in the care and treatment of patients with ARDS, mortality remains unacceptably high and a significant proportion of survivors have persistent pulmonary dysfunction and reduced quality of life. Given the rapidity with which ARDS can evolve, understanding and defining the kinetics and cardinal features of the pathological fibroproliferative response is paramount since the optimal window for therapeutic intervention is most likely to either precede or coincide with the onset of fibroproliferation. Indeed, recent investigations in both ARDS and IPF underscore the difficulty of reversing alreadyestablished fibrosis [24, 83, 90-92]. A better understanding of the fine balance between the physiological and pathological effects of the various profibrotic mediators might allow targeted intervention, for example, with anti-proteinases such as MMP inhibitors in an effort to prevent severe structural damage to the lung and thus tip the scales to facilitate repair to the injured lung [20]. It is our hope that accurate identification of ARDS patients with aberrant fibroproliferation will facilitate the development and use of therapeutic agents that can selectively target molecules involved in the pathological fibroproliferative response (fig. 2). Similarly, accurate identification of patients who will recover quickly and completely is important in order to avoid exposing these individuals to potentially toxic and unnecessary therapies. Strategies that combine clinical characteristics with pathological patterns present on HRCT scans and fibroproliferative markers in BALF or serum may help to identify individuals at risk of poor outcomes that may benefit from targeted therapies.

\section{References}

Ashbaugh DG, Bigelow DB, Petty TL, et al. Acute respiratory distress in adults. Lancet 1967; 2: 319-323.

National Heart, Lung, and Blood Institute Acute Respiratory Distress Syndrome (ARDS) Clinical Trials Network, Rice TW, Wheeler AP, et al. Initial trophic $v s$ full enteral feeding in patients with acute lung injury: the EDEN randomized trial. JAMA 2012; 307: 795-803.

3 ARDS Definition Task Force, Ranieri VM, Rubenfeld GD, et al. Acute respiratory distress syndrome: the Berlin Definition. JAMA 2012; 307: 2526-2533.

4 Mercat A, Richard JC, Vielle B, et al. Positive end-expiratory pressure setting in adults with acute lung injury and acute respiratory distress syndrome: a randomized controlled trial. JAMA 2008; 299: 646-655.

5 The Acute Respiratory Distress Syndrome Network. Ventilation with lower tidal volumes as compared with traditional tidal volumes for acute lung injury and the acute respiratory distress syndrome. N Engl J Med 2000; 342: 1301-1308.

6 National Heart, Lung, and Blood Institute Acute Respiratory Distress Syndrome (ARDS) Clinical Trials Network, Wiedemann HP, Wheeler AP, et al. Comparison of two fluid-management strategies in acute lung injury. N Engl J Med 2006; 354: 2564-2575.

7 Bienvenu OJ, Colantuoni E, Mendez-Tellez PA, et al. Depressive symptoms and impaired physical function after acute lung injury: a 2-year longitudinal study. Am J Respir Crit Care Med 2012; 185: 517-524.

8 Herridge MS, Cheung AM, Tansey CM, et al. One-year outcomes in survivors of the acute respiratory distress syndrome. N Engl J Med 2003; 348: 683-693.

9 Herridge MS, Tansey CM, Matté A, et al. Functional disability 5 years after acute respiratory distress syndrome. N Engl J Med 2011; 364: 1293-1304.

10 Heyland DK, Groll D, Caeser M. Survivors of acute respiratory distress syndrome: relationship between pulmonary dysfunction and long-term health-related quality of life. Crit Care Med 2005; 33: 1549-1556.

11 Masclans JR, Roca O, Muñoz X, et al. Quality of life, pulmonary function, and tomographic scan abnormalities after ARDS. Chest 2011; 139: 1340-1346. 
12 Orme J Jr, Romney JS, Hopkins RO, et al. Pulmonary function and health-related quality of life in survivors of acute respiratory distress syndrome. Am J Respir Crit Care Med 2003; 167: 690-694.

13 Wilcox ME, Patsios D, Murphy G, et al. Radiologic outcomes at 5 years after severe ARDS. Chest 2013; 143: 920-926.

14 Jones PW, Forde Y. St George's Respiratory Questionnaire Manual. London, University of London, 2009. Available from: www.healthstatus.sgul.ac.uk/sgrq/sgrq-downloads

15 McHorney CA, Ware JE Jr, Lu JF, et al. The MOS 36-item Short-Form Health Survey (SF-36): III. Tests of data quality, scaling assumptions, and reliability across diverse patient groups. Med Care 1994; 32: 40-66.

16 Desai SR, Wells AU, Rubens MB, et al. Acute respiratory distress syndrome: CT abnormalities at long-term followup. Radiology 1999; 210: 29-35.

17 Nöbauer-Huhmann IM, Eibenberger K, Schaefer-Prokop C, et al. Changes in lung parenchyma after acute respiratory distress syndrome (ARDS): assessment with high-resolution computed tomography. Eur Radiol 2001; 11: 2436-2443.

18 Burnham EL, Hyzy RC, Paine R 3rd, et al. Chest CT features are associated with poorer quality of life in acute lung injury survivors. Crit Care Med 2013; 41: 445-456.

19 Paine R 3rd, Standiford TJ, Dechert RE, et al. A randomized trial of recombinant human granulocyte-macrophage colony stimulating factor for patients with acute lung injury. Crit Care Med 2012; 40: 90-97.

20 Matthay MA, Ware LB, Zimmerman GA. The acute respiratory distress syndrome. J Clin Invest 2012; 122: 2731-2740.

21 Matthay MA, Zemans RL. The acute respiratory distress syndrome: pathogenesis and treatment. Annu Rev Pathol 2011; 6: 147-163.

22 Ware LB, Matthay MA. The acute respiratory distress syndrome. N Engl J Med 2000; 342: 1334-1349.

23 Zemans RL, Colgan SP, Downey GP. Transepithelial migration of neutrophils: mechanisms and implications for acute lung injury. Am J Respir Cell Mol Biol 2009; 40: 519-535.

24 Davey A, McAuley DF, O'Kane CM. Matrix metalloproteinases in acute lung injury: mediators of injury and drivers of repair. Eur Respir J 2011; 38: 959-970.

25 González-López A, Astudillo A, García-Prieto E, et al. Inflammation and matrix remodeling during repair of ventilator-induced lung injury. Am J Physiol Lung Cell Mol Physiol 2011; 301: L500-L509.

26 Lee WL, Downey GP. Leukocyte elastase: physiological functions and role in acute lung injury. Am J Respir Crit Care Med 2001; 164: 896-904.

27 Dos Santos CC. Advances in mechanisms of repair and remodelling in acute lung injury. Intensive Care Med 2008; 34: 619-630.

28 Madtes DK, Rubenfeld G, Klima LD, et al. Elevated transforming growth factor- $\alpha$ levels in bronchoalveolar lavage fluid of patients with acute respiratory distress syndrome. Am J Respir Crit Care Med 1998; 158: 424-430.

29 Chesnutt AN, Kheradmand F, Folkesson HG, et al. Soluble transforming growth factor- $\alpha$ is present in the pulmonary edema fluid of patients with acute lung injury. Chest 1997; 111: 652-656.

30 Snyder LS, Hertz MI, Peterson MS, et al. Acute lung injury. Pathogenesis of intraalveolar fibrosis. J Clin Invest 1991; 88: 663-673.

31 Tager AM, LaCamera P, Shea BS, et al. The lysophosphatidic acid receptor LPAl links pulmonary fibrosis to lung injury by mediating fibroblast recruitment and vascular leak. Nat Med 2008; 14: 45-54.

32 White KE, Ding Q, Moore BB, et al. Prostaglandin E2 mediates IL-1 $\beta$-related fibroblast mitogenic effects in acute lung injury through differential utilization of prostanoid receptors. J Immunol 2008; 180: 637-646.

33 Chandel NS, Budinger GR, Mutlu GM, et al. Keratinocyte growth factor expression is suppressed in early acute lung injury/acute respiratory distress syndrome by smad and c-Abl pathways. Crit Care Med 2009; 37: 1678-1684.

34 Yi ES, Williams ST, Lee H, et al. Keratinocyte growth factor ameliorates radiation- and bleomycin-induced lung injury and mortality. Am J Pathol 1996; 149: 1963-1970.

35 Verghese GM, McCormick-Shannon K, Mason RJ, et al. Hepatocyte growth factor and keratinocyte growth factor in the pulmonary edema fluid of patients with acute lung injury. Biologic and clinical significance. Am J Respir Crit Care Med 1998; 158: 386-394.

36 Fahy RJ, Lichtenberger F, McKeegan CB, et al. The acute respiratory distress syndrome: a role for transforming growth factor- $\beta 1$. Am J Respir Cell Mol Biol 2003; 28: 499-503.

Wynn TA. Integrating mechanisms of pulmonary fibrosis. J Exp Med 2011; 208: 1339-1350.

38 Rock JR, Barkauskas CE, Cronce MJ, et al. Multiple stromal populations contribute to pulmonary fibrosis without evidence for epithelial to mesenchymal transition. Proc Natl Acad Sci USA 2011; 108: E1475-E1483.

39 Chapman HA. Epithelial-mesenchymal interactions in pulmonary fibrosis. Annu Rev Physiol 2011; 73: 413-435.

40 Schrimpf C, Duffield JS. Mechanisms of fibrosis: the role of the pericyte. Curr Opin Nephrol Hypertens 2011; 20: 297-305.

41 Phillips RJ, Burdick MD, Hong K, et al. Circulating fibrocytes traffic to the lungs in response to CXCL12 and mediate fibrosis. J Clin Invest 2004; 114: 438-446.

42 Pelosi P, Rocco PR. Effects of mechanical ventilation on the extracellular matrix. Intensive Care Med 2008; 34: 631-639.

43 Tremblay LN, Miatto D, Hamid Q, et al. Injurious ventilation induces widespread pulmonary epithelial expression of tumor necrosis factor- $\alpha$ and interleukin-6 messenger RNA. Crit Care Med 2002; 30: 1693-1700.

44 Tremblay LN, Slutsky AS. Ventilator-induced lung injury: from the bench to the bedside. Intensive Care Med 2006; 32: 24-33.

45 Bachofen M, Weibel ER. Structural alterations of lung parenchyma in the adult respiratory distress syndrome. Clin Chest Med 1982; 3: 35-56.

46 Tomashefski JF Jr. Pulmonary pathology of acute respiratory distress syndrome. Clin Chest Med 2000; 21: 435-466.

47 Rocco PR, Dos Santos C, Pelosi P. Lung parenchyma remodeling in acute respiratory distress syndrome. Minerva Anestesiol 2009; 75: 730-740.

48 Keane MP, Belperio JA, Moore TA, et al. Neutralization of the CXC chemokine, macrophage inflammatory protein-2, attenuates bleomycin-induced pulmonary fibrosis. J Immunol 1999; 162: 5511-5518.

49 Ou XM, Li WC, Liu DS, et al. VEGFR-2 antagonist SU5416 attenuates bleomycin-induced pulmonary fibrosis in mice. Int Immunopharmacol 2009; 9: 70-79. 
Hamada N, Kuwano K, Yamada M, et al. Anti-vascular endothelial growth factor gene therapy attenuates lung injury and fibrosis in mice. J Immunol 2005; 175: 1224-1231.

51 Maitre B, Boussat S, Jean D, et al. Vascular endothelial growth factor synthesis in the acute phase of experimental and clinical lung injury. Eur Respir J 2001; 18: 100-106.

52 Thickett DR, Armstrong L, Millar AB. A role for vascular endothelial growth factor in acute and resolving lung injury. Am J Respir Crit Care Med 2002; 166: 1332-1337.

53 Abadie Y, Bregeon F, Papazian L, et al. Decreased VEGF concentration in lung tissue and vascular injury during ARDS. Eur Respir J 2005; 25: 139-146.

54 Parikh SM, Mammoto T, Schultz A, et al. Excess circulating angiopoietin-2 may contribute to pulmonary vascular leak in sepsis in humans. PLoS Med 2006; 3: e46.

55 Clark JG, Milberg JA, Steinberg KP, et al. Type III procollagen peptide in the adult respiratory distress syndrome. Association of increased peptide levels in bronchoalveolar lavage fluid with increased risk for death. Ann Intern Med 1995; 122: 17-23.

56 Farjanel J, Hartmann DJ, Guidet B, et al. Four markers of collagen metabolism as possible indicators of disease in the adult respiratory distress syndrome. Am Rev Respir Dis 1993; 147: 1091-1099.

57 Meduri GU, Tolley EA, Chinn A, et al. Procollagen types I and III aminoterminal propeptide levels during acute respiratory distress syndrome and in response to methylprednisolone treatment. Am J Respir Crit Care Med 1998; 158: $1432-1441$.

58 Bulpa PA, Dive AM, Mertens L, et al. Combined bronchoalveolar lavage and transbronchial lung biopsy: safety and yield in ventilated patients. Eur Respir J 2003; 21: 489-494.

59 Patel SR, Karmpaliotis D, Ayas NT, et al. The role of open-lung biopsy in ARDS. Chest 2004; 125: 197-202.

60 Papazian L, Doddoli C, Chetaille B, et al. A contributive result of open-lung biopsy improves survival in acute respiratory distress syndrome patients. Crit Care Med 2007; 35: 755-762.

61 Krein PM, Sabatini PJ, Tinmouth W, et al. Localization of insulin-like growth factor-I in lung tissues of patients with fibroproliferative acute respiratory distress syndrome. Am J Respir Crit Care Med 2003; 167: 83-90.

62 Marshall RP, Bellingan G, Webb S, et al. Fibroproliferation occurs early in the acute respiratory distress syndrome and impacts on outcome. Am J Respir Crit Care Med 2000; 162: 1783-1788.

63 Budinger GR, Chandel NS, Donnelly HK, et al. Active transforming growth factor- $\beta 1$ activates the procollagen I promoter in patients with acute lung injury. Intensive Care Med 2005; 31: 121-128.

64 Synenki L, Chandel NS, Budinger GR, et al. Bronchoalveolar lavage fluid from patients with acute lung injury/acute respiratory distress syndrome induces myofibroblast differentiation. Crit Care Med 2007; 35: 842-848.

65 de Pablo R, Monserrat J, Reyes E, et al. Sepsis-induced acute respiratory distress syndrome with fatal outcome is associated to increased serum transforming growth factor $\beta-1$ levels. Eur J Intern Med 2012; 23: 358-362.

66 Quesnel C, Nardelli L, Piednoir P, et al. Alveolar fibroblasts in acute lung injury: biological behaviour and clinical relevance. Eur Respir J 2010; 35: 1312-1321.

67 Quesnel C, Piednoir P, Gelly J, et al. Alveolar fibrocyte percentage is an independent predictor of poor outcome in patients with acute lung injury. Crit Care Med 2012; 40: 21-28.

68 Nakayama Y, Kon S, Kurotaki D, et al. Blockade of interaction of $\alpha 9$ integrin with its ligands hinders the formation of granulation in cutaneous wound healing. Lab Invest 2010; 90: 881-894.

69 Mineo G, Ciccarese F, Modolon C, et al. Post-ARDS pulmonary fibrosis in patients with H1N1 pneumonia: role of follow-up CT. Radiol Med 2012; 117: 185-200.

70 Steinberg KP, Mitchell DR, Maunder RJ, et al. Safety of bronchoalveolar lavage in patients with adult respiratory distress syndrome. Am Rev Respir Dis 1993; 148: 556-561.

71 Moeller A, Gilpin SE, Ask K, et al. Circulating fibrocytes are an indicator of poor prognosis in idiopathic pulmonary fibrosis. Am J Respir Crit Care Med 2009; 179: 588-594.

72 Vij R, Noth I. Peripheral blood biomarkers in idiopathic pulmonary fibrosis. Transl Res 2012; 159: $218-227$.

73 Barnett N, Ware LB. Biomarkers in acute lung injury - marking forward progress. Crit Care Clin 2011; 27: 661-683.

74 Calfee CS, Ware LB, Glidden DV, et al. Use of risk reclassification with multiple biomarkers improves mortality prediction in acute lung injury. Crit Care Med 2011; 39: 711-717.

75 Barlo NP, van Moorsel CH, Ruven HJ, et al. Surfactant protein-D predicts survival in patients with idiopathic pulmonary fibrosis. Sarcoidosis Vasc Diffuse Lung Dis 2009; 26: 155-161.

76 Gattinoni L, Caironi P, Cressoni M, et al. Lung recruitment in patients with the acute respiratory distress syndrome. N Engl J Med 2006; 354: 1775-1786.

77 Ichikado K, Suga M, Müller NL, et al. Acute interstitial pneumonia: comparison of high-resolution computed tomography findings between survivors and nonsurvivors. Am J Respir Crit Care Med 2002; 165: 1551-1556.

78 Ichikado K, Suga M, Muranaka H, et al. Prediction of prognosis for acute respiratory distress syndrome with thinsection CT: validation in 44 cases. Radiology 2006; 238: 321-329.

79 Ichikado K, Muranaka H, Gushima Y, et al. Fibroproliferative changes on high-resolution CT in the acute respiratory distress syndrome predict mortality and ventilator dependency: a prospective observational cohort study. BMJ Open 2012; 2: e000545.

80 Chung JH, Kradin RL, Greene RE, et al. CT predictors of mortality in pathology confirmed ARDS. Eur Radiol 2011; 21: 730-737.

81 Grieser C, Goldmann A, Steffen IG, et al. Computed tomography findings from patients with ARDS due to Influenza A (H1N1) virus-associated pneumonia. Eur J Radiol 2012; 81: 389-394.

82 Meduri GU, Headley AS, Golden E, et al. Effect of prolonged methylprednisolone therapy in unresolving acute respiratory distress syndrome: a randomized controlled trial. JAMA 1998; 280: 159-165.

83 Steinberg KP, Hudson LD, Goodman RB, et al. Efficacy and safety of corticosteroids for persistent acute respiratory distress syndrome. $N$ Engl J Med 2006; 354: 1671-1684.

84 Meduri GU, Golden E, Freire AX, et al. Methylprednisolone infusion in early severe ARDS: results of a randomized controlled trial. Chest 2007; 131: 954-963.

85 Beyer C, Distler JH. Tyrosine kinase signaling in fibrotic disorders: translation of basic research to human disease. Biochim Biophys Acta 2013; 1832: 897-904.

86 Yamaguchi Y, Takihara T, Chambers RA, et al. A peptide derived from endostatin ameliorates organ fibrosis. Sci Transl Med 2012; 4: 136ra71. 
87 Massagué J. TGF $\beta$ signalling in context. Nat Rev Mol Cell Biol 2012; 13: 616-630.

Biernacka A, Dobaczewski M, Frangogiannis NG. TGF- $\beta$ signaling in fibrosis. Growth Factors 2011; 29: 196-202. Wrighton KH, Lin X, Feng XH. Phospho-control of TGF- $\beta$ superfamily signaling. Cell Res 2009; 19: 8-20.

90 King TE Jr, Albera C, Bradford WZ, et al. Effect of interferon $\gamma-1 \mathrm{~b}$ on survival in patients with idiopathic pulmonary fibrosis (INSPIRE): a multicentre, randomised, placebo-controlled trial. Lancet 2009; 374: 222-228.

91 Raghu G, Brown KK, Bradford WZ, et al. A placebo-controlled trial of interferon $\gamma$ - $1 \mathrm{~b}$ in patients with idiopathic pulmonary fibrosis. $N$ Engl J Med 2004; 350: 125-133.

92 King TE Jr, Brown KK, Raghu G, et al. BUILD-3: a randomized, controlled trial of bosentan in idiopathic pulmonary fibrosis. Am J Respir Crit Care Med 2011; 184: 92-99. 